\title{
Effect of neuromuscular balance on static balance in adult and elderly populations
}

\author{
Ana Paula Santos Tartari ${ }^{1}$, Felipe Figueiredo Moreira ${ }^{1}$, Mário César da Silva Pereira ${ }^{1}$, Ivon Ilvan Kerppers ${ }^{1}$, Francisco \\ José Cridral-Filho ${ }^{2}$, Afonso Shiguemi Inouse Salgado ${ }^{2}$
}

${ }^{1}$ State University of Centro Oeste of Paraná, Guarapuava (PA), Brazil; ${ }^{2}$ State University of Santa Catarina, Palhoça (SC), Brazil.

\section{ABSTRACT}

Background: Postural control has two behavioral goals: postural balance and orientation, which are achieved through a dynamic relationship between sensory information and muscle activity. To maintain body balance, the vestibular, optical and proprioceptive systems must be fully functioning. Neuromuscular balance (NB), developed by François Soulier, is a non-manipulative method that involves applying a soft vibration or a micro-thrust with a small mechanical device to a joint or vertebra in order to induce realignment and readjustment. Objective: To identify the biomechanical effect of NB upon static balance and body oscillations of adults and the elderly through stabilometry. Methodology: In this interventional study, 20 healthy volunteers - 10 young adults (aged between 18 and 30 years old) and 10 elderly (aged between 60 and 80 years old) - were subjected to 10 consecutive weekly NB sessions. Stabilometry was assessed at baseline and after the $10^{\text {th }}$ intervention (center of pressure (COP) behavior parameters: total area in mm2, laterolateral and anteroposterior width in $\mathrm{mm}$ ). Results: Anteroposterior as well as total area oscillations significantly decreased in the adult population $(p<0.0179$ and $p<0.0242$, respectively). Additionally, all parameters were reduced in both groups, although differences between pre and post intervention were not statistically significant. Conclusion: NB effectively decreased anteroposterior and total area oscillations of adult patients, positively contributing to static balance.

Keywords: Neuromuscular Balance; Stabilometry; Postural Balance; Manipulation.

\section{BACKGROUND}

\section{Static balance}

Maintaining an upright position is a complex task that involves a precise relationship between sensory information and motor activity ${ }^{(1)}$. Postural control has two behavioral goals, named postural balance and orientation, which are achieved through a dynamic relationship between sensory information and muscle activity, and can be evaluated using a force platform, such as a baropodometry or stabilometric platform. Postural balance can be defined as the state in which all the forces acting on the body are balanced, allowing control of the projection of the center of mass of the body within the limits of the support base ${ }^{(2,3)}$.

According to Enoka, a system is in mechanical equilibrium when the sum of forces acting on it is equal to zero, however, this is not an easy task when it comes to the human body ${ }^{(4)}$. Barela states that constant oscillations occur even when a person tries to remain standing as stable as possible ${ }^{(1)}$.

In maintaining body balance, the vestibular, optical and the proprioceptive systems must be fully functioning. This information is used by the postural system to provide an internal reference of the environment through the position and movement of body parts. The abundance of this information guarantees the stability of postural control even with the deficiency of one of these systems ${ }^{(3)}$.

Hennemam ${ }^{(5)}$ and Schmidt ${ }^{(6)}$ define the human body posture as the relationship between the head, trunk and limbs, and affirm that the support of the upright posture requires complex neurological coordination, although

mostly reflex. It has been previously shown that spinal misalignment can result in an inadequate posture, which may lead to exacerbated loss of balance and body oscillations $^{(5,6)}$. The therapeutical approach evaluated in the present study, i.e., The Soulier method, was developed to assess body dealignments, identify posture compensations and correct them through non-invasive subtle adjustment techniques.

Intrinsic as well as extrinsic factors constantly affect physiology. Intrinsic factors are related to changes in cellular mechanism, e.g., the aging process, which is triggered by a genetic program that determines the beginning of senescence ${ }^{(7)}$, but may be accelerated by environmentally induced cellular damage accumulated over the years, i.e., extrinsic conditions, such as radiation, altitude, environmental temperature, pollution, smoking, as well as inadequate diet, poor or inadequate living conditions, among other factors ${ }^{(8)}$.

Several changes can contribute to a loss of balance in the elderly population, such as cognitive impairment, musculoskeletal disorders, pain, visual impairment, medications and sarcopenia ${ }^{(9)}$. One way to assess static balance is to use stabilometry, which is the objective study of the body's oscillation during quiet rest, that is, evaluation of posture in the absence of voluntary movements or external disturbances. Stabilometry is usually based on the analysis of the time variant center of pressure (CoP) coordinates during a bipedal or single leg stance $^{(10)}$. 


\section{Neuromuscular Balance}

Neuromuscular balance (NB) was developed in France in 1993 by a physiotherapist named François Soulier, after five years of study, when he discovered a chiropractic technique named "activator methods", which uses a spring-loaded, hand-held mechanical instrument called "activator adjusting instrument" that generates quick, low-force mechanical impulses, with the aim of replacing the chiropractor hands when performing a sacrum manipulative technique ${ }^{(11,10)}$. In sum, NB is a non-manipulative method that involves applying a soft vibration or a micro-thrust with a small mechanical device to a joint or vertebra in order to induce realignment and readjustment ${ }^{(12)}$.

NB is a very common method in physiotherapy, although only a handful of studies have been conducted with this technique. NB starts with the analysis of the length of the lower limbs with the patient in prone position and, if differences between the two limbs are identified, the physiotherapist will search for misalignments or joint blockages by applying manual pressure to specific areas of the body. Treatment is carried out by soft vibration or a micro-thrusts with the "neuromuscular balancer" mechanical device ${ }^{(13)}$.

NB is a stress-free treatment for the patient that plays an important role in the organization of the proprioceptive system and, consequently, in the improvement of postural muscle tone, allowing the body to balance itself, by stimulating subtle postural corrections. This vibrational stimulation is applied upwards according to the imbalances found ${ }^{(13-14)}$.

This technique is designed to correct joint blocks and for tonic readjustments, and can be combined with any other technique, except for massage and electrothermal phototherapy treatments, which must be performed prior to NB, due to reflex information, which is the basis of this technique, and can be compromised if performed after such interventions ${ }^{(14)}$.

\section{Manipulation and C1}

Manipulation can be defined as the passive movement of a vertebra with high speed and low amplitude, within its physiological range of motion and preserving its anatomical integrity. Headaches are commonly treated with chiropractic. In these cases, it is assumed that the normalization of muscle tone and joint mobility of cervical structures, especially the upper cervical segments, leads to a reduction of nociceptive stimuli to the trigeminal nerve nuclei, and consequent relief of painful symptoms in the innervation territory of this nerve ${ }^{(15-18)}$. There is evidence that joint manipulation therapy (JMT) can be effective for the treatment of migraines, tension-type headaches and cervicogenic headaches ${ }^{(16,17)}$, and even in decreasing systolic blood pressure after specific adjustments of the upper cervical vector (atlas) ${ }^{(19)}$. A study showed ${ }^{(20)}$ decrease in pain and increase in mobility of the temporomandibular joint, after high-speed and lowamplitude manipulation (HSLAM) of the $\mathrm{C} 0$ to $\mathrm{C} 3$ region. The misalignment of the atlas can affect the nervous system by changing the weight in the atlanto-occipital joint, thus stimulating articular mechanoreceptors. The resulting reflexes can create a functional inequality in the length of the legs and observable postural asymmetry, thus, leading to a postural imbalance ${ }^{(21,22)}$.

\section{Rhythmic Reflex Massage (RRM)}

Manual therapies, including massage, are well established techniques used to relieve pain and provide relaxation, among many other functions, and which are constantly being perfected ${ }^{(23)}$. Rhythmic reflex massage (RRM) created by François Soulier, based on conventional massage techniques, emphasizes the importance of touch, and the hand as a therapeutic agent for stimulation of self-healing ${ }^{(24)}$.

According to the data previously described, the objective of the present study was to identify the biomechanical effect of NB upon static balance and body oscillations of adults and the elderly through stabilometry.

\section{METHODS \\ Study Ethics}

Interventional research conducted from February to July 2019 at the Clinical School of the Physiotherapy course at UNICENTRO. Before the beginning of the experiment, this study was approved by the University Ethics Committee for Clinical Research (COMEP) / UNICENTRO (protocol number: 138707180.0.0000.0106 / approved number. 3,322,969).

\section{Sample}

Ten (10) young adults (18 to 30 years old) and 10 elderly individuals (60 to 80 years old) voluntarily participated in this study. All participants signed the study informed consent form.

\section{Inclusion criteria}

Patients who accepted to participate in the study on a voluntary and committed basis.

\section{Exclusion criteria}

Patients with neurological or trauma-orthopedic pathologies who presented functional dysfunction.

\section{Assessment tools}

Participants were assessed using stabilometry with a Footwork brand device that features an $490 \mathrm{~mm} \times 490 \mathrm{~mm}$ active surface; dimensions $565 \times 420 \times 25 \mathrm{~mm}$; Weight 3Kg; 4096 (6x6) calibrated capacitors; PC connection: USB2 compatible 1.1; analog conversion: 16 bits; Frequency: $200 \mathrm{~Hz}$; pickup size: 7.62 x $7.62 \mathrm{~mm}$; maximum pressure per pickup: $120 \mathrm{~N} / \mathrm{cm} 2$.

\section{Statistical analysis}

The results were analyzed using GraphPad Prism (version 6.0, La Jolla, California, USA) to determine means, standard deviations and $p$ values. Data were analyzed with unpaired $t$ test, with a confidence level of $95 \%$. Values of $p<0.05$ were considered to be statistic 
ally significant. For the standardization of oscillation values, a calculation was performed for each patient, based on the study by Rocchi and collaborators ${ }^{(25)}$.

\section{Treatment}

After the evaluation, the volunteers were seen once a week, in 20-minute sessions, once a week for 10 consecutive weeks. The NB protocol used in this study consisted of applying the Atlas (C1) Specific Technique, followed by the RRM, and was divided into 5 stages, 1) Examine, test and find the injury, asking the patient to perform a trunk and cervical rotation, examine the fingerto-floor distance, and trunk inclination, to identify which side is less mobile, 2) Identify the side to be corrected, according to the length of the upper limbs 3) Identify the three axes of the injury, which are divided into flexion or cervical extension; inclination and rotation to the right or left side, 4) Correction of Cervical Vertebra 1 (Atlas) (hole in one), which is performed standing up according to the evaluation performed 5) Verification of the result ${ }^{(26)}$.

Afterwards, RRM is applied in three steps: 1) Lying down, 2) Sitting, 3) Standing.

Lying down: In prone position, upper limbs along the body, the therapist's hands descending along the spine to the sacrum, with gentle, regular and light pressures with one hand, meanwhile, the other hand performing knee flexion and extension. All of these phases were first performed passively and then actively, which consisted of the patient performing the movements unassisted ${ }^{(26)}$

Sitting: With one hand, the therapist performs circular movements with the patient's ankle, and with the other hand performs gentle pressure on the lateral regions of the ankle, going up along the tibia, fibula and posterior leg, and performing knee extension and flexion at the same time, while massaging around the knee and moving up the quadriceps ${ }^{(26)}$. Standing: With one hand, the therapist rotates the patient's thumb and wrist, and with the other presses the bones of the carpus, forearm and radial head, continuing in the lateral region of the upper limb (deltoid and upper trapezius) with abduction of shoulder, and finally, palpating the pectoral region associating it with shoulder adduction. All of these phases were first performed passively and then actively, which consisted of the patient performing the movements unassisted ${ }^{(26)}$

\section{RESULTS}

The evaluated outcomes with significantly statistical differences between baseline and postintervention were: Body's center of pressure (CoP) antero-posterior oscillation $(p=0.0179)$ and, Body's center of pressure (CoP) total area oscillation $(\mathrm{p}=$ 0.0242 ) in the group of adults, however, all variables had lower average results compared to pre-treatment, that is, the three oscillations assessed here decreased after treatment. Table 1 shows the $p$ values, the mean body oscillations of adult patients and, in percentage, the difference between pre and post treatment.

Below are the average values for each variable evaluated.

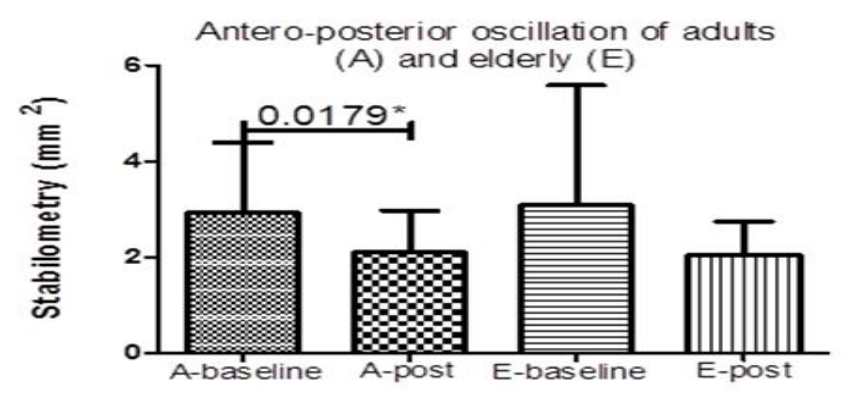

Figure 1. Stabilometric parameter: Body's center of pressure (CoP) antero-posterior oscillation. Each column represents the mean of 10 individuals, and the vertical lines indicate the S.D.M.

*Note: $p<0.05$, when comparing baseline with post-intervention (paired t-test)

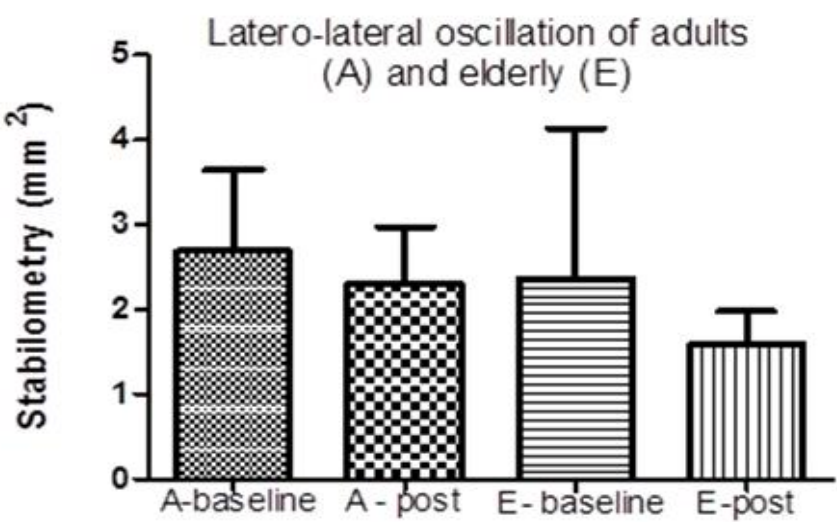

Figure 2. Stabilometric parameter: Body's center of pressure (CoP) latero-lateral oscillation. Each column represents the mean of 10 individuals, and the vertical lines indicate the S.D.M.

Note: $p<0.05$, when comparing baseline with post-intervention (paired t-test)

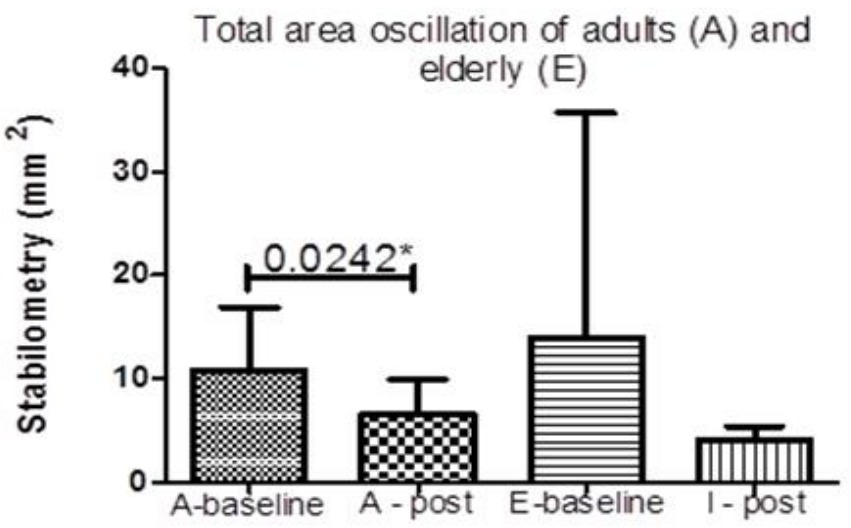

Figure 3. Stabilometric parameter: Body's center of pressure (CoP) total area oscillation. Each column represents the mean of 10 individuals, and the vertical lines indicate the S.D.M.

Note: $p<0.05$, when comparing baseline with post-intervention (paired t-test). 
Table 1. values of means, $p$ and, percentages of the adult group

\begin{tabular}{lcccc}
\hline & P value & Mean (Baseline) & Mean (Post-TTO) \\
\hline $\begin{array}{l}\text { CoP antero-posterior } \\
\text { oscillation }\end{array}$ & $0.0179^{*}$ & 4.958 & 3.563 & $28 \%$ \\
CoP latero-lateral oscillation & 0.1878 & 4.563 & 3.898 & $15 \%$ \\
CoP total area oscillation & $0.0242^{*}$ & 18.47 & 11.23 & $39,2 \%$
\end{tabular}

*Note: Table 1: Stabilometric parameter: anteroposterior oscillation, latero-lateral and total area oscillation, $\mathrm{p}$-value of all variables, before treatment and, after treatment, and finally the percentages referring to the differences of these two parameters (before and after).

Although not statistically significant, all parameters were reduced in the elderly group after 10 treatments, as shown in Table 2.

Table 2. values of means, $p$ and, percentages of the elderly group.

$\mathrm{P}$ value $\quad$ Mean (Baseline) Mean (Post-TTO)
$\%$

\begin{tabular}{lllll}
\hline CoP antero-posterior oscillation & 0.4504 & 5.072 & 3.379 & $50 \%$ \\
CoP latero-lateral oscillation & 0.3270 & 3.920 & 2.641 & $54 \%$ \\
CoP total area oscillation & 0.3691 & 23.20 & 6.836 & $66 \%$
\end{tabular}

"Note: Stabilometric parameter: anteroposterior oscillation, latero-lateral and total area oscillation, $\mathrm{p}$-value of all variables, before treatment and, after treatment, and finally the percentages referring to the differences of these two parameters (before and after).

Results indicate that, in both groups, there was a decrease of the three oscillations evaluated after $10 \mathrm{NB}$ sessions. This data is more significant in CoP total area oscillation in both groups, where in the adult group a

\section{DISCUSSION}

Aquaroli and collaborators ${ }^{(27)}$ applied manual mobilization techniques, cervical traction, pompage, stretching, myofacial inhibition and, finally, joint manipulation in patients with cervical radiculopathy, and the treatment method proved to be effective, reducing analgesia and functional disability.

Teixera $^{(28)}$, carried out a study with seven participants, to evaluate the effect of cervical manipulation (C7) on the balance of asymptomatic individuals, and results showed that manipulation reduced postural oscillations (unipodal balance assessment) during the first two attempts made, reflecting an acute effect. After five minutes this effect tended to gradually disappear. In the present study, with the Atlas Specific treatment, all patients maintained a better static balance after 10 visits, although data was not statistically significant in the elderly group.

Valente ${ }^{(12)}$, analyzed the effects of NB in its first stage, which consisted of assessing the possible existence of joint blocks in the knees, ankles, pelvis, decrease of $39,2 \%$ was observed, and in the elderly group, $71 \%$, that is, these patients had their postural system adjusted after treatment (although data was not statistically significant).

sacrum, pubis, spine, first rib, shoulders, and checking for difference in length in the limbs, followed by correction using the neuromuscular balancer. Results showed the efficiency of this protocol through the distribution of means in the hindfoot, midfoot and forefoot, occasionally decreasing body sway, corroborating with the present study.

Gabardo and Caberlon ${ }^{(28)}$, reported that problems of vertebral instability and its effects may be related to the Atlas. In this context, these authors conducted a study with 15 students and teachers who presented complaints of chronic or acute neck pain, and the reassessment made immediately after NB, showed that there was a statistically significant decrease in pain $(p<0.0001)$, as well as a decrease in local temperature and range of motion, but without statistically significant results.

Neta ${ }^{(11)}$ and Campos ${ }^{(13)}$ carried out similar studies, which consisted of the application of NB in patients with chronic low back pain. Both studies demonstrated that NB increased spine mobility. $100 \%$ of the cases were positive 
in Campos' study after just one application. Although this was not the main objective of the present study, some of the participants reported immediate relaxation, greater flexibility and decreased back pain after the sessions.

Schneider and collaborators ${ }^{(29)}$ and Gorrell and collaborators $^{(30)}$ also carried out similar studies comparing manual and instrumental manipulation in low back and cervical pain, respectively, using the activator methods (activator IV), in both studies there were no statistically significant differences between the techniques.

These effects occur through the stimulation of body proprioceptors that the technique provides, translating into a smaller number of oscillatory movements, promoting a better static balance.

\section{CONCLUSION}

NB effectively decreased anteroposterior and total area oscillations of adult patients, positively contributing to static balance. Although parameters were also reduced in the elderly group, differences between pre and post intervention were not statistically significant.

The technique developed by François Soulier is an innovative, simple, and fast approach, with no adverse effects for patients. Although commonly used, it lacks scientific research.

As improvement of static balance may be an important preventive therapeutic tool in adults and the elderly, a larger size, randomized, placebo-controlled clinical trial with this technique is recommended.

Authors' contributions: All authors contributed equally to the production of the study. Organization and planning of the research project, data collection and text organization; correction of the final version in Portuguese, correction of the final version in English, correction of the statistical analysis.

Financial support: The authors declare that there is no Non-financial competing interests

Conflict of interest: The authors declare that there was no conflict of interests.

\section{REFERENCES}

1. Barela JA. Estratégias de controle em movimentos complexos: ciclo percepção-ação no controle postural. Rev Paul Educ Fís. 2000; (supl.3), 79-88.

2. Meneghetti $\mathrm{CHZ}$. Static balance assessment among children and adolescents with Down syndrome. Rev Brasil Fisiot. 2009;13(3):230-235.

3. Leme GLM, Carvalho IF, Scheicher ME. Melhora do equilíbrio postural em mulheres idosas com o uso de informação sensorial adicional. Fisiot e Pesq. 2017;24(1):68-73.

4. Enoka RM. Bases neuromecânicas da cinesiologia. ed.2. São Paulo: Manolo, 2000.

5. Hennemam E. Fisiolo méd, Rio de Janei: Guanabara Koogan, 1982
6. Schmidt A. Estudo das alterações morfológicas do sistema locomotor em escolares do Ensino Fundamental - faixa etária entre 7 e 14 anos da ambos os sexos do município de Marechal Cândido Rondou, PR - através da avaliação postural computadorizada. UNICAMP, 1999.

7. Cendes IL. Fatores Genéticos e Envelhecimento. Desenvolvimento e Envelhecimento: perspectivas biológicas, psicológicas e sociológicas. SP:Papirus. 2001, 200, 53-60.

8. Calkins E. Geriatria Prática. 2.ed. Rio de Janeiro: Revinter, 1997

9. Cuevas-Trisan R. Balance Problems and Fall Risks in the Elderly. 2017;28(4):727-737.

10. Nagymáté $G$, Orlovits $Z$, Kiss RM. Reliability analysis of a sensitive and independent stabilometry parameter set. Plos One. 2018, 13(4):e0195995.

11. Neta EAF, Leal LPA, Meireles KAD. A efetividade do equilíbrio neuromuscular na dor lombar. Redalyc. 2008;21(3):187-193.

12. Valente MM. Análise baropodométrica e estabilométrica em mulheres jovens antes, durante e após a aplicação do protocolo de base do método de equilíbrio neuromuscular (NB). 2006.

13. Campos TM. Résultat immédiat de l'Equilibration Neuromusculaire (NB) dans le traitement des lombalgies chroniques. Thérapie manuelle. 2005;3: 362-365.

14. Soulier F. Curso de equilíbrio neuromuscular. São Paulo: Arte e Produções, 2006.

15. Bracher EAB, Benedicto CC, Facchinato APA. Quiropraxia. Rev Medicina. 2013;92(3):173-82.

16. Bortolazzo GL. Manipulação de alta velocidade e baixa amplitude. Unip. 2010

17. Woodfield HC, Hasick DG, Becker WJ, Rose MS, Scott JN. Effect of Atlas Vertebrae Realignment in Subjects with Migraine: An Observational Pilot Study. BioMed Resear Intern. 2015;2015:630472.

18. Hall T, Chan HT, Chirstensen L, Odenthal B, Wells C, Robinson K. Efficacy of a C1-C2 self-sustained natural apophyseal glide (SNAG) in the management of cervicogenic headache. J Orthop Sports Phys Ther. 2007;37(3):100-7.

19. Knutson GA. Significant changes in systolic blood pressure post vectored upper cervical adjustment vs resting control groups: A possible effect of the cervicosympathetic and/ or pressor reflex. Journal of Manipulative \& Physiological Therapeutic. 2001;24(2):101-9.

20. Kamonseki DH, Fonseca CL, Souza TP, Zamunér AR, Peixoto BO, Chiao L. Efeito imediato da manipulação thrust aplicada na coluna cervical alta sobre a abertura ativa da boca: ensaio clínico randomizado. J Health Sci Inst. 2012;30(3): 277-80.

21. Crowe $\mathrm{H}$, Kleinman $\mathrm{T}$. Upper cervical influence on the reticular system. Upper Cervical Monograph. $1991 ; 5(1): 12-14$.

22. Knutson GA. abnormal upper cervical joint alignment and the neurologic component of atlas subluxation complex. Chirop Reserch J. 1997;4(1):5-9. 
23. Oliveira TS, Uliano CBO, Carvalho DK. Estudo comparativo sobre os efeitos da massagem reflexa da coluna associada a pedras quentes no alívio da dor e desconforto musculoesquelético dorsal. Unisul, 2017.

24. Machado MP. Méthode François Soulier, quiropraxia instrumental. São Paulo: $1^{\circ}$ ed Andreoli, 2019.

25. Rochi L, Chiari L, Cappello A. Feature selection of stabilometric parameters based on principal component analysis. Medical e Biolog Engin e Comput. 2004;42(1):71-9.

26. Aquaroli RS, Camacho ES, Pimenta L. A terapia manual e a estabilização segmentar no tratamento da radiculopatia cervical. Fisioter mov. 2016;29(1):45-52.
27. Teixera GF. Efeito da manipulação cervical no equilíbrio de indivíduos assintomáticos. Portal da osteopatia, 2016.

28. Gabardo AC, Caberlon CF. Influência imediata da técnica atlas específico de NB na dor cervical. Mét François Soulier quiro instru. 2019;9:178-184.

29. Scheneider MJ, Brach J, Irrgang JJ, Abbott KV, Wisniewski SR, Delitto A. Mechanical versus manual manipulation for low back pain: An observational cohort study. J Manipulat Physiol Ther. 2010;33(3):193-200.

30. Gorrell LM, Beath K, Engel RM. Manual and instrument applied cervical manipulation for mechanical neck pain: a randomized controlled trial. Journ of Manipul and Physio Therapeu. 2016;39(5):319-329. 